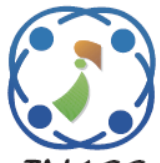

\title{
An Efficient Routing Protocol Using an Improved Distance-Based Broadcasting and Fuzzy Logic System for VANET
}

\author{
Khalid Kandali $^{1 *} \quad$ Hamid Bennis ${ }^{1}$ \\ ${ }^{I}$ IMAGE Laboratory, SCIAM Team, Graduate School of Technology, \\ Moulay Ismail University of Meknes, Morocco \\ * Corresponding author’s Email: kandalikhalid@hotmail.com
}

\begin{abstract}
The high mobility and frequent changes in the network topology remain a great divergence between Vehicular Ad-hoc Network (VANET) and Mobile Ad-hoc Network (MANET). This makes existing routing protocols for MANET imperfect for a VANET environment. In order to ensure good data transmission between vehicles, it is mandatory to improve these protocols to this new environment. Ad-hoc On-demand Distance Vector (AODV) is one of the most proposed reactive protocols in the literature for MANET, but its direct application in VANET returns poor performance results. In this paper, we propose an Efficient Routing Protocol using an improved Distance-Based Broadcasting and Fuzzy Logic System (ERPFL), which is an extension of the AODV routing protocol. In the first, the proposed scheme uses an improved Distance-Based Broadcasting method in route discovery process. Each vehicle selects the most suitable neighbours to send a route request, using different mobility parameters such as distance, velocity and direction. Then, the destination vehicle selects the best route from the most reliable routes received, using the Fuzzy Logic System. Link Expiration Time and Link Reliability Model are considered input metrics for this system. The simulation results show that ERPFL saves network bandwidth resources which contributes to a higher data transmission ratio. In addition, our proposed protocol efficiently distributes data packets, decreases the hop count and reduces network routing time.
\end{abstract}

Keywords: VANET, Routing protocol, AODV, Distance-based broadcasting, Fuzzy logic, Link expiration time, Link reliability model.

\section{Introduction}

Recently, Vehicular Ad-hoc Network have become a promising field of research to improve the Intelligent Transportation System (ITS). This technology has attracted great attention from ITS designers, automakers, private research agencies, and university research laboratories [1, 2]. Indeed, VANET networks provide a long list of applications that constitute Intelligent Transportation Systems [3]. These applications are classified into security applications and comfort applications. Safety applications aim to avoid accidents and improve road safety, such as optimizing paths between vehicles, monitoring traffic and preventing collisions. While comfort applications aim to improve the level of passenger comfort, such as parking spaces and restaurants, weather forecasting, the nearest hotels and petrol stations $[4,5]$.

In VANET network, vehicles can communicate with each other without any infrastructure required, or each vehicle can randomly join or leave the network $[6,7]$. These vehicles are generally equipped with network interfaces called On Board Unit (OBU) [8]. The VANET network is a self-configured and self-maintained network that does not require any centralized authority. Each node of the network can send, receive and relay packets to the other nodes of the network [9].

The VANET network being considered as a subclass of the MANET networks [10-12], several researches and studies have shown the weakness of the routing protocols designed for MANET when applied directly to the vehicular environment [13], due to the very growing number of vehicles on the 
roads, highly mobility and frequent disconnections $[14,15]$. Indeed, the major issue of VANET remains in the time and the reliability of routing information through different ITS applications [16].

In the literature, several works have recently been done to optimize the existing routing protocols and adapt them to the VANET characteristics. There are several types of protocols, but reactive routing protocols are still highly recommended and popular [17]. This type of protocol discovers the routes and maintains them only on demand [18]. The most studied and optimized routing protocol in the literature is Ad-hoc On-demand Distance Vector (AODV) [19, 20]. In fact, this protocol has several drawbacks, notably at the level of the Route Discovery Phase [21]. In this process, the source node generates a large number of Route Request (RREQ) packets when it wants to find a better routing path to the destination node, which increases the network overhead [22]. In addition, when the destination node receives several routes, it searches for the best route based on the number of nodes in each routing path [23]. This factor is not sufficient to select the best route, especially in a VANET networks.

Broadcasting in VANET poses more challenges due to the mobility and density of vehicles. Due to the mobility aspect, there is no single optimal scheme for all scenarios. Indeed, the quality of a VANET network lies in the ability to broadcast effectively packets among network vehicles. Thus, efficient packet broadcasting is a key element to obtain high performance in VANET.

In this context, this paper proposes an improved Distance-Based Broadcasting method in route discovery process. Each vehicle selects the most suitable neighbours to send a route request, using different mobility parameters such as distance, velocity and direction. Each vehicle sends or forwards a certain number of RREQ packets, which does not exceed a certain threshold. The threshold will be defined by each vehicle according to the characteristics of the network. In addition, the destination vehicle selects the best route from the most reliable routes received, by using the Fuzzy Logic System. Link Expiration Time (LET) and Link Reliability Model (REL) are considered input metrics for this system.

The methodology of this paper is recapitulated as follows:

- At first, we explain how each node calculates and updates the threshold value, taking into account the characteristics of the network. This value determines the maximum number of RREQ packets broadcasted by each node in Route Discovery Process. We avoid considering a fixed threshold value, so as not to have a risk disturbing the Route Discovery Phase, when we have a high-density network.

- Then, we present the method to select a number of neighbouring nodes that does not exceed a certain threshold, to broadcast RREQ packets. The selection is made by calculating the link weight of each neighbouring node.

- Then, to choose the most stable and reliable route between the nodes, a Fuzzy Logic system is introduced, when the input metrics are LET and REL.

- Finally, an extensive simulation is carried out by modifying the number of vehicles to verify the effectiveness of the proposed method, and compare it with several methods proposed in the literature.

Briefly, the main contributions of this paper are:

- Proposing an improved Distance-Based Broadcasting method in Route Discovery Process.

- Selection of the reliable route to transmit data in VANET using the Fuzzy Logic. LET and REL are the input metrics for this system.

- A comparative evaluation of performance of ERPFL, CALAR-DD [24] and M-GEDIR [29] in terms of Packet Delivery Ratio, Average Endto-End delay and hop count.

Thus, in the rest of the paper, section 2 presents several routing protocols proposed in the literature. Then, section 3 provides an overview of the Fuzzy Logic system used in this paper. In section 4, we illustrate the proposed approach. Then, section 5 details the simulation process, and displays the results. Finally, we will conclude this paper in section 6.

\section{Related works}

Various researches have been proposed to correct the aforementioned shortcomings on AODV in order to improve this routing protocol and adapt it to VANET.

In this section, we present the main modifications recently made on the AODV routing protocol to improve and adapt it to VANET.

A cache agent-based location aided routing using distance and direction (CALAR-DD) was introduced in [24], which combines geocasting and position routing. In the first step, CALAR-DD selects the next hop vehicle to forward the packet until it arrived at the expected region. Whereas, the second step consists in using cache agent geocasting in the expected region, to select and forward the packets to the destination. However, the authors have not 
proposed a method for selecting the most reliable route by the destination vehicle.

In order to improve vehicular communication in urban environment and computational resources, in [25], the authors presented a position-based routing protocol named Improved Geographic Routing (IGR), which depends on the distance to the destination and the vehicle density of each street to select the junction. And to transmit a data between two junctions, IGR uses an improved greedy forwarding, which take into account the link error rate in the path selection. However, the proposed method did not take into account the velocity of the vehicles. This parameter represents a major challenge in the VANET environment.

In [26], the authors proposed two algorithms to design a new routing protocol called Improved Directional LAR routing protocol. The first algorithm is Select Next Forwarder (SNF), which considers the nodes adjacent to the border area that has minimum distance. While, the second algorithm is called Packet Forwarding Algorithm (PFA), which is used to broadcast Routing packet to the neighbouring nodes. This approach aims to find the best next-hop forwarder node in forward area. The main objective of which is to reduce unnecessary transmissions. As the selection of the best path to transmit the data is a challenge in VANET networks, the proposed method did not address this point. Also, the mathematical model used during the data transmission, did not take into account other mobility parameters such as the velocity and direction of the vehicles.

[27] introduced a reliable path selection and packet forwarding routing protocol (RPSPF), in order to create a suitable route between vehicles to send data. RPSPF considers the connectivity and the shortest appropriate distance based on several intersections. And in order to avoid packet loss during packet forwarding, the proposed routing protocol applies a novel reliable packet forwarding technique between intersections. The selection of next hop in the proposed method is based on the partitioning of road networks instead of being based on mobility parameters and the quality of connections between vehicles.

This paper [28] introduced a fuzzy assisted position-based routing protocol (FPBR-DTN) for $\mathrm{V} 2 \mathrm{~V}$ communication, the main objective of which is to ameliorate greedy routing and keep away absurd node for routing. However, a neighbouring node that has the highest chance value is been selected for the greedy forwarding. This value is calculated by applying Fuzzy Logic and different parameters, such as the number of neighbours, the direction of neighbouring vehicles, their speed and their distance form a destination. Then the greedy mode, perimeter and DTN are considered as the main modes of FPBRDTN. This greedy scheme enhances the efficiency of routing protocol. However, the authors have not proposed a method for selecting the most reliable route by the destination vehicle. In addition, other parameters like Link Expiration Time can be used as input parameters for the Fuzzy Logic System.

In [29], the author introduced a Multi-metric geographic routing technique (M-GEDIR), in order to find vehicles for the next hop in dynamic forwarding regions. M-GEDIR has taken into account the future position of vehicles, critical area vehicles at the border of transmission range, and the strength of the received signal. In NHV selection, MGEDIR uses a maximum weighting factor. However, when selecting the next hop, this work does not take into account the quality of the link, which could augment the possibility of link failure.

A Fuzzy Logic-Based Geographic Routing for Urban VANET in [30], in order to improve road safety and transportation capacity. This approach associated several metrics, such as direction of vehicle position, link quality and achievable throughput, to find an appropriate next-hop node for packet forwarding. The Fuzzy logic system coordinated and evaluated the metrics considered. While, the algorithm used ETX for the appreciation of the quality of the link. However, the authors used the Fuzzy Logic system for each node before transmitting the packets, which causes significant delays, in particular for the high network densities and the low mobility of the vehicles.

In [31], a novel geographic routing protocol called LTQGR was proposed for an urban VANET. This protocol was based on a new routing metric called Link Transmission Quality (LTQ), in order to select the routing path with the lowest aggregated weights for the transmission data. The transmission quality of the link took into account the cost and the reliability of transmission. However, this work does not consider other mobility parameters such as direction and speed in the design of the new metric concerning the quality of transmission link.

In [17], a new version of the AODV routing protocol was been proposed. This new version was based on two strategies. The first was to improve HELLO packets and control packets by sharing information from neighbouring node in the network. This strategy has made it possible to reduce the network overhead. The second strategy was based on an alternative routing option to deliver data packets in a short time. Generally, the authors have sought through this paper to improve the packet delivery ratio by reducing the use of bandwidth. This paper did 
not use new metrics to test the stability of the routes between the nodes. The proposed algorithm took into consideration Hop Number as basic metric to select the best and the alternative paths.

[32] suggested a cross-layer optimized positionbased routing protocol for urban VANET. This approach aimed to facilitate cooperation in the network and to solve misbehaving nodes which knowingly drop packets by presenting a social aspect to its design. However, it was recommended that nodes with a close favourable social relationship will be net forwarder nodes, otherwise nodes with explicit distrust connections were rated favoured in the network.

In order to minimize the impact of the position error, due to various factors, such as signal fading, tunnels and line-of-sight, the paper [33] designed a predicted Position Based Routing Protocol (PPRP) for VANET. This approach used the Kalman Filter (KF) to guess the vehicle location based on the previous and current location. The selection of the next section in the proposed method is based on the position of the vehicles instead of being based on the mobility parameters and the quality of the connections between the vehicles.

A distance and direction-based location aided on multi hop routing protocol (DD-LAR) for the city traffic scenario was suggested in [34], where the vehicle which had a minimum angle with maximum distance covered from the source was selected for the Next Hop Vehicle. But DD-LAR was interested in relative distance to select the vehicle which are in the source transmission range in case there is no vehicle to choose according to the minimum angle with maximum distance. Therefore, this approach aimed to reduce the hop count in routing process. The authors did not introduce the speed parameter to examine the performance on path duration and hop count metric.

[35] proposed a cache agent-based geocasting (CAG). The mains additional concepts of which were Re-caching and full range radio transmissions assimilated with the Connectivity Assurance Algorithm (CAA). The proposed protocol aimed to reduce identical packets, hop count, packet loss and hop-to-hop communications using CAA. Then the cache packet will not be transmitted if there has been no guaranteed packet confirmation. However, to reduce the number of hops in route delivery, CAG used full radio power.

The paper [18] presented a Pro-AODV routing protocol based on information in the routing table in order to minimize congestion in VANET networks. It was based on the size of the routing table for each node and did not require the execution of any additional logic. Thanks to this, the proposed protocol can find routes to congestion-free destinations, which has contributed to higher delivery and a lower drop ratio. This proposed approach aimed to improve the packet delivery ratio, average end-toend, drop ratio and control overhead. This paper used probability-based broadcasting to solve the problems associated with the simple flooding method. Each node has received a predetermined probability to rebroadcast packets. However, there was a risk that some nodes might not receive routing or data packets.

To summarize this section, these proposed works present one or more of the following limitations: (i) The selection of next hop and the most stable path is based on the partitioning of road networks instead of being based on mobility parameters, such as position, speed and mobility; (ii) Several studies do not provide complete approaches, which must combine the selection of next hop and the most stable path, in order to propose an efficient routing protocol.; (iii) The choice of suitable input parameters for the Fuzzy Logic System must take into consideration the quality of the links between the vehicles. However, our proposed scheme provides a complete routing protocol for VANET. This approach is based on an improved Distance-Based Broadcasting, which takes into account several mobility parameters, and a method of selecting the most stable path using the Fuzzy Logic System, whose input parameters are linked to the quality of the links between the vehicles.

\section{Fuzzy logic system}

Fuzzy logic is a theory formalized by professor Lotfi Zadeh in 1965 . This system is considered to be an artificial intelligence tool, based on mathematical logic. It extends classic Boolean logic with partial truth values, which are real numbers between 0 and 1 . Fuzzy logic consists of a few basic concepts, such as: fuzzy set, if-then rules, and membership function, and it has four steps, as shown in Fig. 1: Fuzzification, rule base, inference engine and defuzzification.

The fuzzification step converts any type of fuzzy input into fuzzy sets. It assigns an integer number of a system to fuzzy sets with a certain degree of membership between 0 and 1 . However, each value corresponds to the degree of uncertainty that the value is included in the set. In this step, the fuzzy sets are illustrated with words that we can think of in a linguistically natural process. The rule base includes a limited number of rules which are used to link the fuzzy input variables to the fuzzy output variables. An inference engine includes a rules database, and it is used to process the production of fuzzy decisions. 


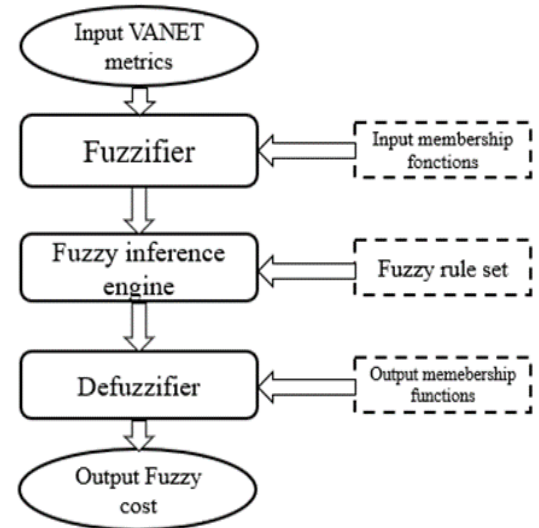

Figure. 1 Fuzzy logic system for ERPFL

When, the defuzzification step consists in obtaining continuous variable from the fuzzy truth values [36].

\section{Proposed approach}

Our main intention in this paper is to improve the Route Discovery Phase to increase the quality of routing in VANET. This work proposes an improved Distance-Based Broadcasting, by using the mobility information available on the network such as the position, the speed and the direction of the nodes. And then to select the most stable route, using the Fuzzy Logic System. We introduce a first factor called Threshold Number TN. TN limits the number of routing packets sent by each node in the network. Each vehicle selects the most suitable neighbours to send a route request, using different mobility parameters such as distance, velocity and direction. The threshold value will be updated by each node according to the density of the network. The second part of the proposed approach aims to select the suitable route between the source node and the destination node. In route discovery process, each node that receive RREQ packet, calculates LET and REL metrics using the position, speed and direction parameters that are inserted into RREQ. Upon receipt of a route request by the destination node, it immediately sends LET and REL to the Fuzzy Logic System, in order to calculate the fuzzy cost of each route. Finally, the route with the higher cost function will be selected as the appropriate route to send RREP packet to the source node.

\subsection{Selection of nodes with stable weight-based in route discovery process}

\subsubsection{Threshold number}

In order to update the threshold number $\mathrm{TN}$ to transmit the routing packets, each node will need several parameters like, the old stored threshold value, the accumulated number of neighbours and hop count
Table 1. Notations and descriptions

\begin{tabular}{|c|l|}
\hline Notation & \multicolumn{1}{c|}{ Description } \\
\hline$T N$ & $\begin{array}{l}\text { Threshold number of packets sent by } \\
\text { each node. }\end{array}$ \\
\hline$T N_{\text {old }}$ & Old threshold value stored in each node. \\
\hline$T N B$ & $\begin{array}{l}\text { Accumulated number of neighbouring } \\
\text { nodes. }\end{array}$ \\
\hline$N B$ & $\begin{array}{l}\text { Number of neighbouring nodes for each } \\
\text { node. }\end{array}$ \\
\hline$H C$ & $\begin{array}{l}\text { Value stored in routing packets, } \\
\text { indicates the number of hops. }\end{array}$ \\
\hline$L W$ & Link Weight value. \\
\hline$D$ & Distance between two nodes. \\
\hline$V_{i}$ & Velocity of node $i$. \\
\hline$\theta$ & Angle of direction. \\
\hline
\end{tabular}

value of the route. This information will be available for each node upon receipt of each routing packet.

The different notations used in this section are mentioned in Table 1.

The following formula is used to calculate the new threshold value:

$$
T N=\alpha \times T N_{\text {old }}+\beta \times\left(\frac{T N B}{H C}\right)
$$

Where $\alpha+\beta=1$.

TNB is calculated by using the following formula:

$$
T N B=\sum N B_{i}
$$

The source node adds the total number of the neighbouring nodes TNB to the RREQ packet before sending it. Each node that receives this packet, calculates the number of its neighbours NB, and will update the cumulative total number of neighbours. Using Eq. (1), the node updates TN with the new calculated value. The same procedure is repeated when the destination node sends the RREP packet to the source node.

In fact, each node updates its threshold value according to the number of nodes connected. This method is described in the algorithm 1 .

\subsubsection{Link weight}

The Link Weight LW is a metric used to select the most suitable neighbouring nodes, in order to send route request.

Let N1 and N2 be two nodes with a speed V1, V2, and direction $\theta 1, \theta 2$ respectively. $\mathrm{LW}$ is calculated by Eq. (3):

$$
L W=W_{D} \times D+W_{V} \times\left|V_{1}-V_{2}\right|+W_{\theta} \times\left|\theta_{1}-\theta_{2}\right|
$$




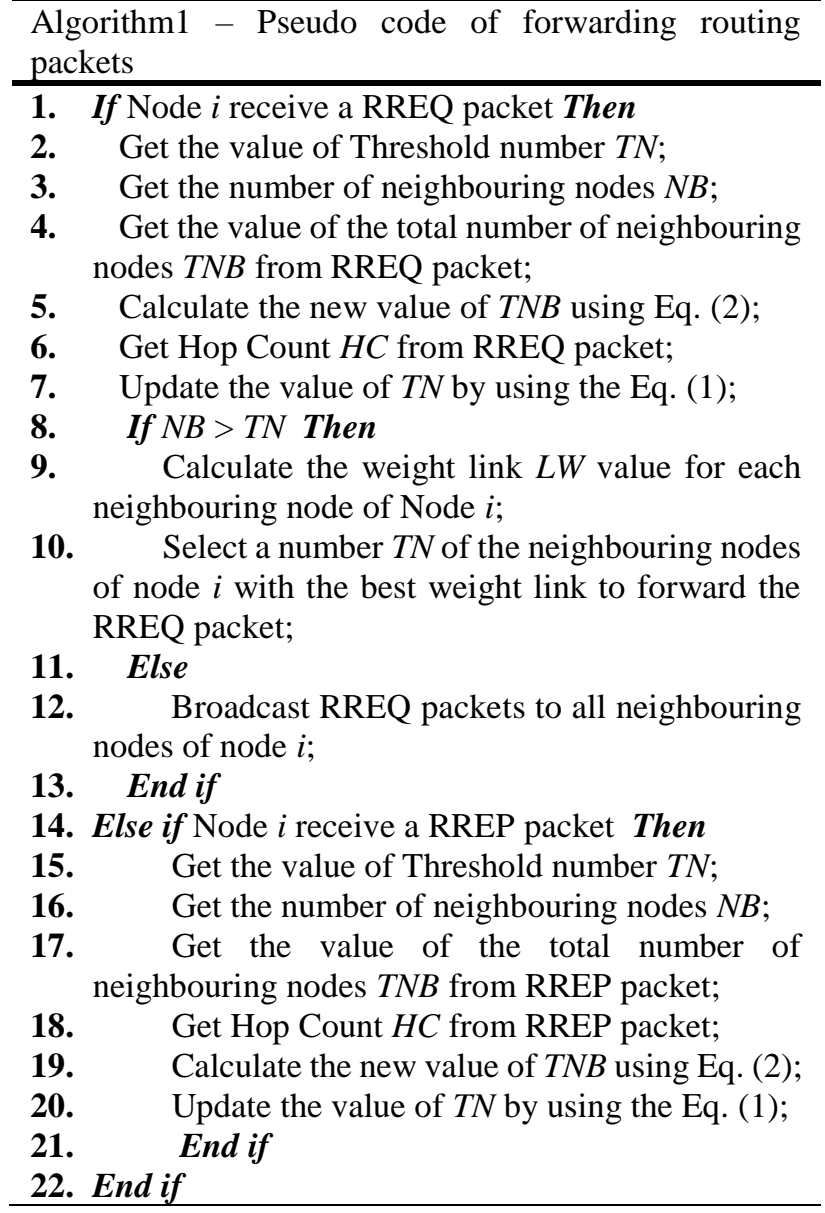

$W_{D}, W_{V}$, and $W_{\theta}$ are the weight factor of distance, velocity and direction respectively.

Thus, the links which carry the least weight are considered more stable links.

Upon receipt of a route request, each node calculates the number of its neighbour nodes NB. If this number is less than the threshold value TN, the node broadcasts the packet RREQ to all its neighbours; if the number of neighbours $\mathrm{NB}$ is greater than $\mathrm{TN}$, the node selects the most suitable neighbours which have the least link weight to send route request.

This process is described in the following pseudo code.

\subsection{Selection of the most stable route using fuzzy logic system}

\subsubsection{Input VANET metrics}

\section{a. Link Expiration Time}

In VANET networks characterized by high mobility of vehicles, it is strongly recommended to take into account the durability of the links when creating routing paths. A long duration of the link between the nodes makes the routing paths more efficient and functional for a long time. Routing paths
Table 2. Notations and descriptions

\begin{tabular}{|c|l|}
\hline Notation & \multicolumn{1}{|c|}{ Description } \\
\hline$L E T$ & Link Expiration Time. \\
\hline$R E L$ & Link Reliability. \\
\hline$T_{p}$ & $\begin{array}{l}\text { Time interval for a link between two } \\
\text { vehicles to be available }\end{array}$ \\
\hline$R$ & Transmission range. \\
\hline$L$ & Distance between two vehicles. \\
\hline$\Delta v$ & $\begin{array}{l}\text { Velocity difference between two } \\
\text { vehicles. }\end{array}$ \\
\hline
\end{tabular}

based on more durable links increase data transmission performance, and reduce network maintenance costs. We suggest LET to test how long two vehicles remain connected. Thus, links with a high LET value are considered the most appropriate.

The different notations used in this section are mentioned in Table 2.

Let $N_{1}$ and $N_{2}$ be two position nodes $\left(x_{1}, y_{1}\right),\left(x_{2}\right.$, $\left.y_{2}\right)$. These two nodes are in movement with a speed $V_{l}$ and $V_{2}$, and with a direction angle $\theta_{1}$ and $\theta_{2}$ respectively. We assume that the two nodes have the same transmission range $R$. As indicated in [37], the LET between the two nodes $N_{1}$ and $N_{2}$ is given by Eq. (4):

$$
L E T=\frac{-(a b+c d)+\sqrt{\left(a^{2}+c^{2}\right) R^{2}-(a d-b c)^{2}}}{a^{2}+c^{2}}
$$

Where:

$$
\begin{aligned}
& a=V_{1} \cos \theta_{1}-V_{2} \cos \theta_{2} \\
& b=x_{1}-x_{2} \\
& c=V_{1} \sin \theta_{1}-V_{2} \sin \theta_{2} \\
& d=y_{1}-y_{2}
\end{aligned}
$$

\section{b. Link Reliability Model}

The reliability of the links REL between two vehicles in VANET is an important parameter in assessing the stability of the system. As noted in [15], this metric shows the probability that the transferability of data between two vehicles remain available for a specific period of time. This probability is calculated by Eq. (5):

$$
\begin{gathered}
R E L=P\{\text { To continue to be availabe until } t+ \\
\left.T_{p} \mid \text { available at } t\right\}
\end{gathered}
$$

Position, speed and direction information are used to calculate REL value of the link $l$. This information is obtained using the GPS installed on each vehicle.

Let $C_{i}$ and $C_{j}$ two vehicles in movement. The value of $T_{p}$ shows the time interval for a link between these two vehicles to be available, and it is calculated according to the following possible cases:

Both vehicles have the same direction: 


$$
T_{p}=\left\{\begin{array}{ll}
\frac{2 R-L_{i j}}{\left|V_{i}-V_{j}\right|} & \text { if } V_{j}>V_{i} \\
\frac{R-L_{i j}}{\left|V_{i}-V_{j}\right|} & \text { if } V_{i}>V_{j}
\end{array} \text { with } V_{j} \neq V_{i}\right.
$$

The two vehicles are moving in a different direction:

$$
T_{p}=\left\{\begin{array}{c}
\frac{R+L_{i j}}{\left|V_{i}+V_{j}\right|} \\
C_{i} \text { and } C_{j} \text { are moving toward } \\
\text { each other } \\
\frac{R-L_{i j}}{\left|V_{i}+V_{j}\right|} \\
C_{i} \text { and } C_{j} \text { are moving away from } \\
\text { each other }
\end{array}\right.
$$

With $R$ is the transmission range and $L_{i j}$ is the distance between the two vehicles. It is calculated by Eq. (8):

$$
L_{i j}=\sqrt{\left(x_{i}-x_{j}\right)^{2}-\left(y_{i}-y_{j}\right)^{2}}
$$

To calculate $R E L$, we use the following error function:

$$
\operatorname{erf}(x)=\frac{2}{\sqrt{\pi}} \int_{0}^{x} e^{-t^{2}} d t
$$

This function is also called GAUSS error function. It is generally used in the fields of statistics and probability.

From the previous formula, we can calculate $R E L$ by Eq. (10):

$$
R E L=\operatorname{erf}\left[\frac{\left(\frac{2 R}{t}-\mu_{\Delta V}\right)}{\sigma_{\Delta V} \sqrt{2}}\right]-\operatorname{erf}\left[\frac{\left(\frac{2 R}{t+T_{p}}-\mu_{\Delta V}\right)}{\sigma_{\Delta V} \sqrt{2}}\right],
$$

Where $\mu$ and $\sigma^{2}$ indicate the average value and the variance of velocity, $\Delta v=\left|v_{2}-v_{1}\right|$ is the velocity difference between two vehicles.

\subsubsection{Fuzzification}

The membership functions are systematized into three variables: Low, Medium and High. The membership function of LET and REL are mentioned in Fig. 2 and Fig. 3. Indeed, these metrics are disposed as arranged at the Fuzzy Logic System.

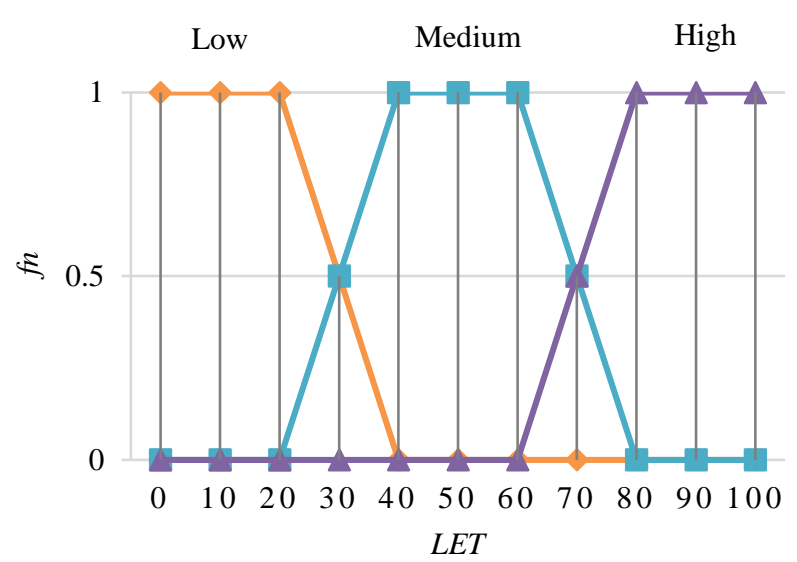

Figure. 2 Membership function of LET

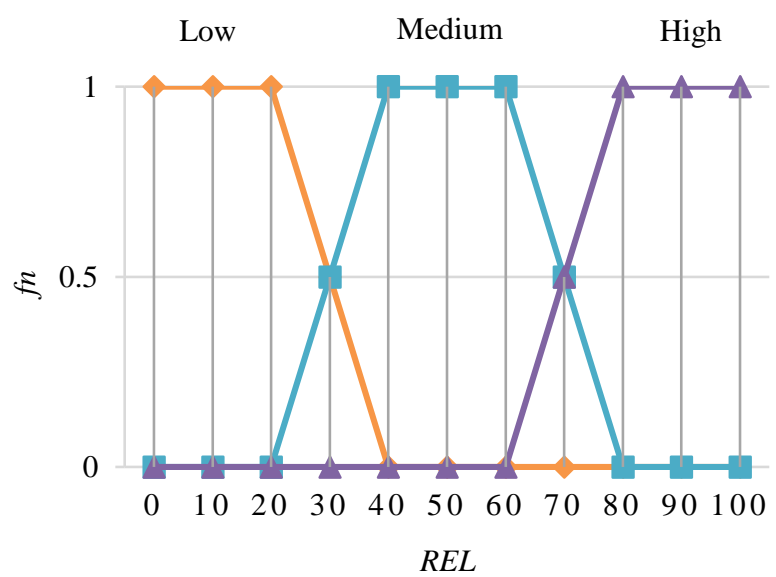

Figure. 3 Membership function of REL

\subsubsection{IF-THEN rules}

As mentioned in Table 3, fuzzy rules are introduced based on the IF-THEN rules. These rules are pre-defined and combined for mapping fuzzy values, and they are used to calculate the link fuzzy cost for each route.

Table 3. Rule base

\begin{tabular}{|l|l|l|l|}
\hline No. & LET & REL & Fuzzy cost \\
\hline 1 & Low & Low & Very bad \\
\hline 2 & Low & Medium & Bad \\
\hline 3 & Low & High & Acceptable \\
\hline 4 & Medium & Low & Bad \\
\hline 5 & Medium & Medium & Acceptable \\
\hline 6 & Medium & High & Good \\
\hline 7 & High & Low & Acceptable \\
\hline 8 & High & Medium & Good \\
\hline 9 & High & High & Perfect \\
\hline
\end{tabular}


Table 4. Route request packet of ERPFL

\begin{tabular}{|c|c|c|c|}
\hline Type & Flags & Reserved & Hop count \\
\hline \multicolumn{4}{|c|}{ RREQ ID } \\
\hline \multicolumn{4}{|c|}{ Destination IP address } \\
\hline \multicolumn{4}{|c|}{ Destination sequence number } \\
\hline \multicolumn{4}{|c|}{ Original IP address } \\
\hline \multicolumn{4}{|c|}{ Original sequence number } \\
\hline \multicolumn{4}{|c|}{ TNB } \\
\hline \multicolumn{4}{|c|}{ Position } \\
\hline \multicolumn{4}{|c|}{ Velocity } \\
\hline \multicolumn{4}{|c|}{ Direction } \\
\hline \multicolumn{4}{|c|}{ LET } \\
\hline \multicolumn{4}{|c|}{ REL } \\
\hline
\end{tabular}

\subsubsection{Defuzzification}

The defuzzification steps take into account the output membership function and its degree to find a precise output value from the fuzzy space. In this step, we use a Center Of Area (COA) method which can be performed via the following equation:

$$
\text { fuzzy }_{\text {cost }}=\frac{\sum_{i=1}^{n} X_{i} \cdot \mu_{C}\left(X_{i}\right)}{\sum_{i=1}^{n} \mu_{C}\left(X_{i}\right)}
$$

Where, $\mu \mathrm{c}$ is a discrete membership function, $\mathrm{Xi}$ is defined as the fuzzy variable, and $n$ is the number of rules.

This step converts the Fuzzy Logic output function to absolute numbers. This number is used to select the most reliable route between the source node and the destination node for transmitting data.

Thus, the route discovery process starts when a source node has to send a data packets to a destination node, and it does not find a route in its routing table. In fact, it broadcasts the route request packet to neighbouring nodes. The format of the route request packet is described in Table 4 . When a neighbouring node receives a route request packet, it creates a reverse route to the source node if it does not already exist in its routing table. A reverse route is mandatory to return response packets to the source node. If the receiving node is the destination node, it simply generates and sends the RREP packet.

If a node receives a RREQ packet, and it is not the destination node, and it already has a route in the routing table to the source node, it checks the LET and REL values of the RREQ packet, and calculates a new LET and REL values. If LET and REL calculated is greater than LET and REL presented in RREQ, the new values are updated in RREQ. Finally, when a destination node receives RREQ, its checks the LET and REL values, and sends them to Fuzzy logic to calculate fuzzy cost of the route. The route with a higher fuzzy cost will be selected to transmit RREP to the source node.

\section{Simulation and results}

\subsection{Simulation setup}

To evaluate the performance of our proposed routing protocol, we used the NS2 network simulator. It is free software and is widely used in academic research. It is implemented in $\mathrm{C}++$ with optional Python bindings, which allows to write simulation scripts in $\mathrm{C}++$ or python. The simulation is carried out on a network of size $1500 \mathrm{~m} \times 1500 \mathrm{~m}$. We used the Random Waypoint Mobility model, where each node starts from any point. The nodes move in the same direction or in a different direction. The simulation lasted 1400 seconds.

The simulation process demonstrates the quality of the proposed approach in terms of Packet Delivery Ratio (PDR), Average End to End Delay (E2ED) and Average number of Hop Count. In which, we varied the number of vehicles from 40 to 200 , and we varied the speed of vehicles from $10 \mathrm{Km} / \mathrm{h}$ to $50 \mathrm{Km} / \mathrm{h}$, in order to assess the efficiency of ERPFL in a network many connected nodes, and high mobility. The performance of proposed protocol was compared with CALAR-DD [24] and M-GEDIR [29]. All other parameters are described in Table 5.

After several tests, we obtained the best results when we start the simulation with the following parameters: $\alpha=0.3 ; \beta=0.7 ; W_{D}=0.2 ; W_{V}=0.3 ; W_{\theta}$ $=0.5$.

\subsection{Simulation results}

\subsubsection{Packet delivery ratio}

Fig. 4 shows the impact of density on Packet Delivery Ratio for ERPFL, CALAR-DD [24] and MGEDIR [29]. The proposed protocol achieves a higher PDR than CALAR-DD and M-GEDIR. The PDR value of CALAR-DD and M-GEDIR decreases from $94 \%$ and $86 \%$ to $88 \%$ and $80 \%$ respectively, when the number of nodes increases. While the PDR of ERPFL keeps a value greater than $87 \%$, even if the number of nodes increases. While Fig. 5 illustrates the impact of speed on PDR of ERPFL, CALAR-DD [24] and M-GEDIR [29]. The PDR of ERPFL retains a value higher than $80 \%$ for the different speeds considered, while the PDR of CALAR-DD and MGEDIR decreases when the speed of the vehicles increases. The PDR loses its value up to $60 \%$ for 
Table 5. Simulation parameters

\begin{tabular}{|l|l|}
\hline \multicolumn{1}{|c|}{ Parameter } & \multicolumn{1}{c|}{ Value } \\
\hline NS-2 version & NS-2.35 \\
\hline Simulation time & $1400 \mathrm{~s}$ \\
\hline Area size & $1500 \mathrm{~m} \times 1500 \mathrm{~m}$ \\
\hline Number of nodes & $40,80,120,160,200$ \\
\hline Mobility model & Random Way Point \\
\hline Minimum speed & $10 \mathrm{Km} / \mathrm{h}$ \\
\hline Maximum speed & $50 \mathrm{Km} / \mathrm{h}$ \\
\hline Transmission range & $250 \mathrm{~m}$ \\
\hline MAC protocol & IEEE $802.11 \mathrm{p}$ \\
\hline Channel bandwidth & $10 \mathrm{MHz}$ \\
\hline Propagation model & Two-ray ground \\
\hline Traffic type & Constant bit rate (CBR) \\
\hline Data rate & $6 \mathrm{Mbps}$ \\
\hline Packet size & 512 bytes \\
\hline Protocols used & $\begin{array}{l}\text { ERPFL, CALAR-DD, M- } \\
\text { GEDIR }\end{array}$ \\
\hline$\alpha, \beta$ & $0.3,0.7$ \\
\hline $\mathrm{W}_{\mathrm{D}}, \mathrm{W}_{\mathrm{v}}, \mathrm{W}_{\theta}$ & $0.2,0.3,0.5$ \\
\hline
\end{tabular}

CALAR-DD and 50\% for M-GEDIR. This efficiency is due to the ability of ERPFL to successfully use speed, direction and position information in the selection of neighbouring nodes to send routing packets. Therefore, ERPFL selects more stable and reliable paths to transmit data from source to destination using Fuzzy Logic System. This saves network bandwidth resources and contributes to a higher data transmission ratio.

\subsubsection{Average end-to-end delay}

Fig. 6 illustrates the progression of the average End-to-end delay for ERPFL, CALAR-DD [24] and M-GEDIR [29]. The results show that our proposed protocol requires less propagation time whether for low or high density and mobility. However, the Average E2ED of CALAR-DD increases from 0,5s to $1,3 \mathrm{~s}$, and the Average E2ED of M-GEDIR increases from $0,7 \mathrm{~s}$ to $1,8 \mathrm{~s}$ when density becomes the main challenge. While ERPFL maintains a high efficiency and the delay does not exceed 0,4s. Fig. 7 shows the impact of velocity on average End-to-End delay for ERPFL, CALAR-DD [24] and M-GEDIR [29]. The delay of ERPFL does not exceed 0,5s despite the increase in vehicle speed, but the delay of CALAR-DD and M-GEDIR increases respectively by $0,5 \mathrm{~s}$ and $0,7 \mathrm{~s}$ to $1,3 \mathrm{~s}$ and $1,7 \mathrm{~s}$. The destination node in our proposed algorithm quickly finds the most reliable route using LET and REL as the input metric to Fuzzy Logic System, and sends the RREP packets along the suitable route that has the higher fuzzy cost value. By calculating the value of the fuzzy

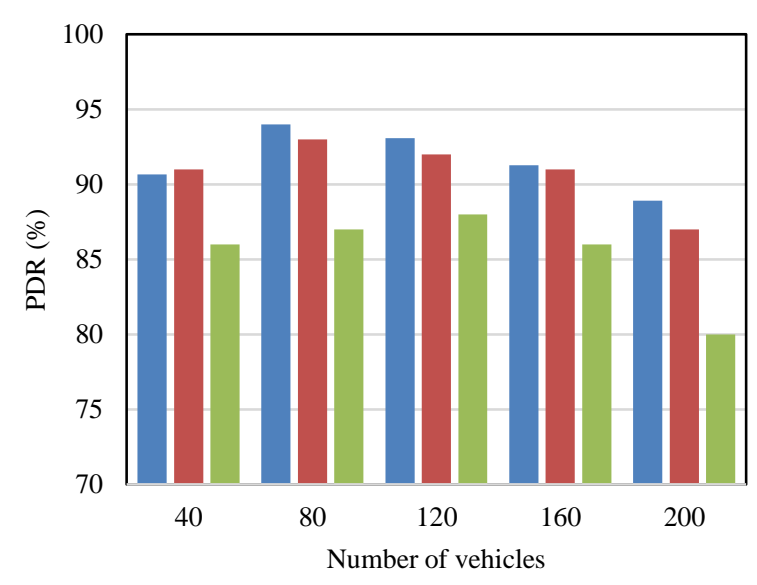

$\square$ ERPFL $\square$ CALAR-DD $\square$ M-GEDIR

Figure. 4 PDR vs number of vehicles

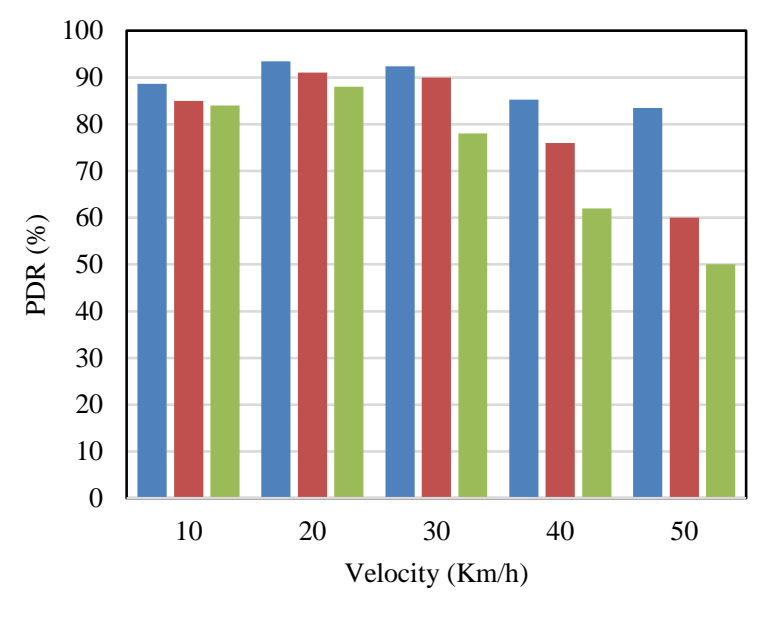

ERPFL $\square$ CALAR-DD $=$ M-GEDIR

Figure. 5 PDR vs velocity

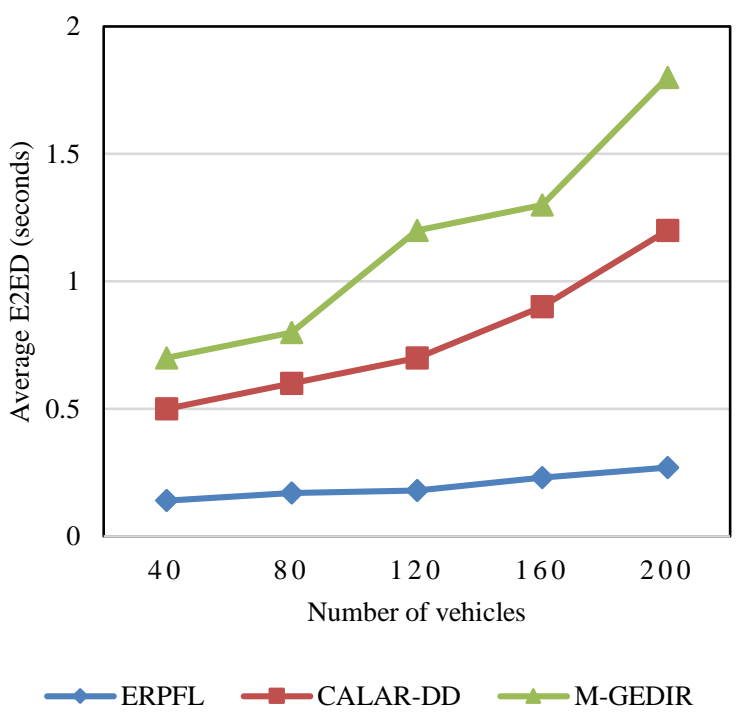

Figure. 6 Average E2ED vs number of vehicles 


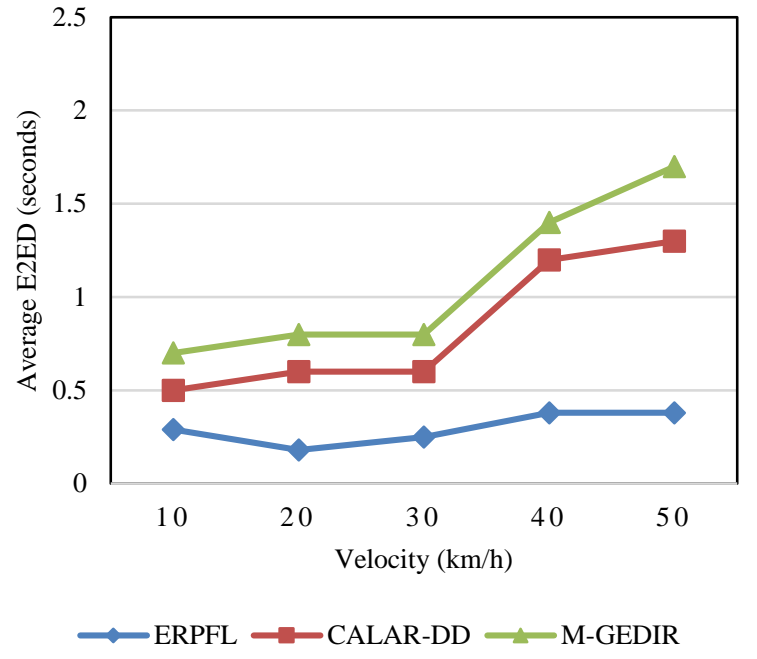

Figure. 7 Average E2ED vs velocity

cost of each route using REL and LET, ERPFL shows its success in a high-density network and high mobility to find a stable and shorter route, and with a maximum lifetime.

\subsubsection{Average number of hop count}

Fig. 8 shows a comparison between ERPFL, CALAR-DD [24] and M-GEDIR [29], in terms of average number of Hop Count. Indeed, ERPFL and CALAR-DD generally have the lowest value of number of hops counts than M-GEDIR. The hop count of ERPFL decreases form $0,7 \%$ to $0,2 \%$, as the number of vehicles increases. So that the hop count of CALAR-DD starts better with $0,6 \%$ and decreases to $0,2 \%$. But the hop count of M-GEDIR is higher than ERPFL and CALAR-DD. While Fig. 9 shows the impact of velocity on Hops Counts for ERPFL, CALAR-DD [24] and M-GEDIR [29]. The hop count of ERPFL reaches a better value than CALAR-DD and M-GEDIR. It starts with $0,7 \%$ and increases to $1,6 \%$, while the hop count of CALAR-DD and MGEDIR increases from $0,6 \%$ and $0,8 \%$ to $2 \%$ and $2,7 \%$ respectively, when the vehicle speed increases. The fact that the nodes in ERPFL know the position, speed and direction of their neighbouring nodes. The proposed protocol uses a minimum number of hops counts in route discovery process. Therefore, decreasing the Hops Counts reduces congestion, efficiently distributes data packets and reduces network routing time.

\section{Conclusion}

In this paper, we have introduced an efficient routing protocol using Fuzzy Logic for VANET, called ERPFL. This approach is based in the first step

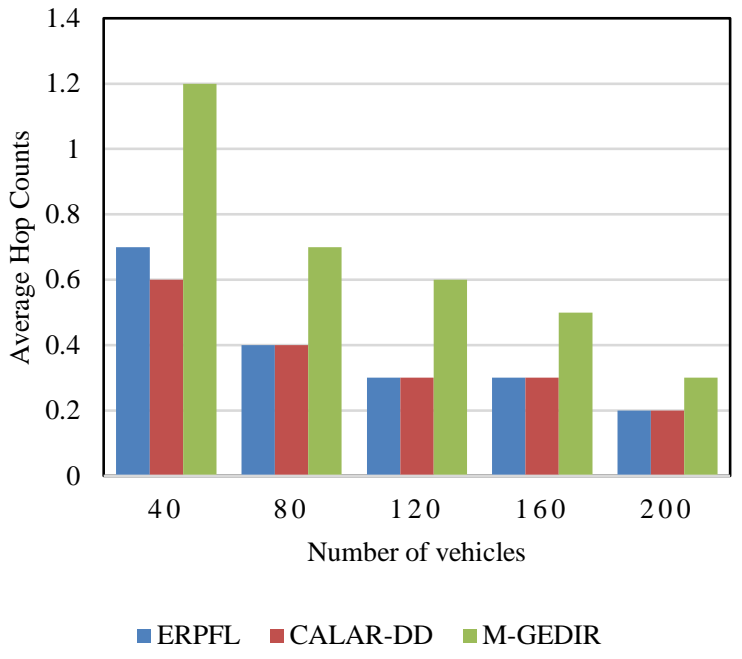

Figure. 8 Average hop count vs number of vehicles

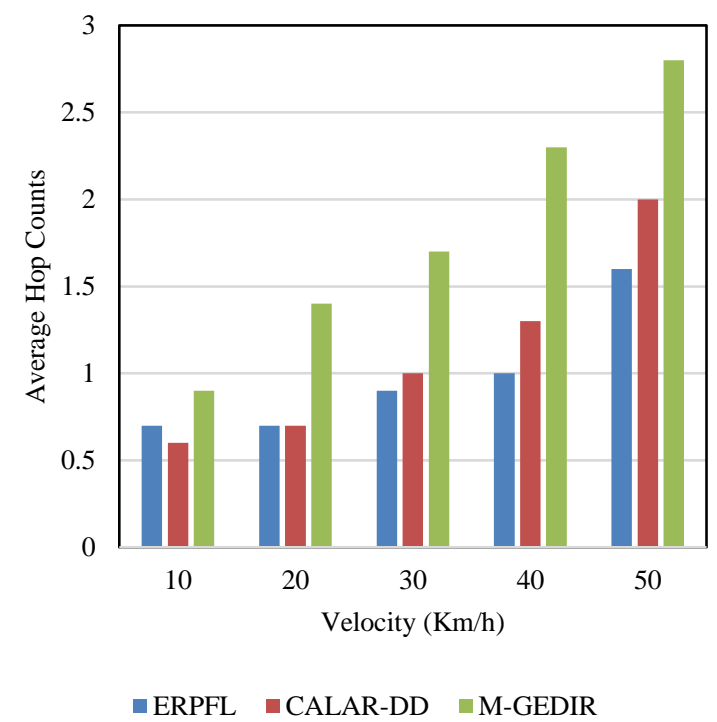

Figure. 9 Average hop count vs velocity

on a method for selecting the nodes to send routing packets in Route Discovery Process, each node chooses a limited number of neighbours to send the RREQ packet, instead of broadcasting the packet to all neighbouring nodes. In the second step, we used a Fuzzy Logic System to select the best route to transmit data. Indeed, ERPFL calculates a fuzzy cost for each route, using a Fuzzy Logic System, where the inputs of this system are LET and REL. The route between nodes with a higher fuzzy cost is selected to transmit data. ERPFL aims to reduce bandwidth consumption, reduce packet routing costs and ensure a permanent data transmission in a VANET.

We have evaluated and compared the ERPFL proposed routing protocol with CALAR-DD [24] and M-GEDIR [29]. The simulation results show that ERPFL succeeded in using speed, direction and position to select the reliable neighbouring nodes to 
send routing packets. Therefore, ERPFL saves network bandwidth resources, which contributes to a higher data transmission ratio. In addition, using Fuzzy Logic and parameters such as LET and REL as input metrics, ERPFL shows its efficiency in a highdensity network and high mobility to find a stable and shorter route, and with a maximum lifetime. Finally, our proposed protocol decreases the Hop Count and reduces congestion. In this sense, ERPFL efficiently distributes data packets and reduces network routing time.

\section{Conflicts of Interest}

The authors declare no conflict of interest.

\section{Author Contributions}

Conceptualization, Kandali and Bennis; methodology, Kandali and Bennis; software, Kandali; validation, Kandali and Bennis; formal analysis, Kandali and Bennis; writing - original draft preparation, Kandali and Bennis; writing - review and editing, Kandali and Bennis; visualization, Bennis; supervision, Bennis; project administration, Bennis.

\section{References}

[1] F. Mekelleche and H. Hafid, "Towards the Development of Vehicular Ad-Hoc Networks (VANETs): Challenges and Applications", IoT and Cloud Computing Advancements in Vehicular Ad-Hoc Networks, pp. 21-47, 2020.

[2] F. Goudarzi, H. Asgari, and H. S. Al-Raweshidy, "Traffic-Aware VANET Routing for City Environments-A Protocol Based on Ant Colony Optimization", IEEE Systems Journal, Vol. 13, No. 1, pp. 571-581, 2019.

[3] D. Lin, J. Kang, A. Squicciarini, Y. Wu, S. Gurung, and O. Tonguz, "MoZo: A Moving Zone Based Routing Protocol Using Pure V2V Communication in VANETs", IEEE Transactions on Mobile Computing, Vol. 16, No. 5, pp. 1357-1370, 2017.

[4] C. Ghorai, and I. Banerjee, "A constrained Delaunay Triangulation based RSUs deployment strategy to cover a convex region with obstacles for maximizing communications probability between V2I", Vehicular Communications, Vol. 13, pp. 89-103, 2018.

[5] S. Al-Sultan, M. M. Al-Doori, A. H. Al-Bayatti, and $\mathrm{H}$. Zedan, "A comprehensive survey on vehicular Ad Hoc network", Journal of Network and Computer Applications, Vol. 37, pp. 380-392, 2014.
[6] S. Alam, S. Sulistyo, W. Mustika, and R. Adrian, "Utility-Based Horizontal Handover Decision Method for Vehicle-to-Vehicle Communication in VANET", International Journal of Intelligent Engineering and Systems, Vol. 13, No. 2, 2020.

[7] K. C. Lee, U. Lee, and M. Gerla, "Survey of Routing Protocols in Vehicular Ad Hoc Networks", Advances in Vehicular Ad-Hoc Networks: Developments and Challenges, pp. 149-170, 2010.

[8] R. Adrian, S. Sulistyo, W. Mustika, S. Alam, "ABNC: Adaptive Border Node Clustering Using Genes Fusion Based on Genetic Algorithm to Support the Stability of Cluster in VANET", International Journal of Intelligent Engineering and Systems, Vol. 13, No. 1, 2020.

[9] K. Kandali, and H. Bennis, "Performance Assessment of AODV, DSR and DSDV in an Urban VANET Scenario", In: Proc. of Advanced Intelligent Systems for Sustainable Development (AI2SD'2018), M. Ezziyyani, Éd. Cham: Springer International Publishing, Vol. 915, pp. 98-109, 2019.

[10] K. Kandali, H. Bennis, and M. Benyassi, "A Novel Route Discovery Mechanism Based on Neighborhood Broadcasting Methods in VANET", In: Proc. of Ezziyyani M. (eds) Advanced Intelligent Systems for Sustainable Development (AI2SD'2019). AI2SD 2019. Lecture Notes in Networks and Systems, Springer, Cham, Vol. 92, 2020.

[11] H. Fatemidokht and M. K. Rafsanjani, "F-Ant: an effective routing protocol for ant colony optimization based on fuzzy logic in vehicular ad hoc networks", Neural Computing and Applications, Vol. 29, No. 11, pp. 1127-1137, 2018.

[12] G. Li, L. Boukhatem, and J. Wu, "Adaptive Quality-of-Service-Based Routing for Vehicular Ad Hoc Networks with Ant Colony Optimization", IEEE Transactions on Vehicular Technology, Vol. 66, No. 4, pp. 3249-3264, 2017.

[13] A. Naushad, G. Abbas, Z.H. Abbas, and L. Jiao, "A novel faster failure detection strategy for link connectivity using Hello messaging in mobile ad hoc networks", International Journal of Ad Hoc and Ubiquitous Computing, Vol. 32, No. 2, pp. 75-98, 2019.

[14] S. A. Soleymani, A. H. Abdullah, M. H. Anisi, A. Altameem, W. H. Hasan, S. Goudarzi, S. Mandala, Z. B. Razak, and N. M. Noor, "BRAIN-F: Beacon Rate Adaption Based on Fuzzy Logic in Vehicular Ad Hoc Network", 
International Journal of Fuzzy Systems, Vol. 19, No. 2, pp. 301-315, 2017.

[15] M. H. Eiza, T. Owens, and Q. Ni, "Secure and Robust Multi-Constrained QoS Aware Routing Algorithm for VANETs", IEEE Transactions on Dependable and Secure Computing, Vol. 13, No. 1, pp. 32-45, 2016.

[16] F. Azzali, O. Ghazali, and M. H. Omar, "Fuzzy Logic-based Intelligent Scheme for Enhancing QoS of Vertical Handover Decision in Vehicular Ad-hoc Networks", In: Proc. of IOP Conf. Series: Materials Science and Engineering, Vol. 226, pp. 012081, 2017.

[17] S. Mittal, S. Bisht, K. C. Purohit, and A. Joshi, "Improvising-AODV routing protocol by modifying route discovery mechanism in VANET", In: Proc. of 2017 3rd International Conf. on Advances in Computing, Communication \& Automation (ICACCA) (Fall), Dehradun, pp. 1-5, 2017.

[18] T. Kabir, N. Nurain, and M. H. Kabir, "ProAODV (Proactive AODV): Simple modifications to AODV for proactively minimizing congestion in VANETs", In: Proc. of 2015 International Conf. on Networking Systems and Security (NSysS), Dhaka, pp. 1-6, 2015.

[19] N. Javaid, A. Bibi, S. N. Mohammad, Z. A. Khan, and N. Alrajeh, "Towards optimising routing overhead in wireless multi-hop networks", International Journal of Ad Hoc and Ubiquitous Computing, Vol. 19, No. 1, 2, pp. 418, 2015.

[20] S. M. Khan, R. Nilavalan, and A. F. Sallama, "A Novel Approach for Reliable Route Discovery in Mobile Ad-Hoc Network", Wireless Personal Communications, Vol. 83, No. 2, pp. 15191529, 2015.

[21] M. Wei, K. Wang, and Y. Hsieh, "A reliable routing scheme based on vehicle moving similarity for VANETs", In: Proc. of TENCON 2011 - 2011 IEEE Region 10 Conf. Bali, Indonesia, pp. 426-430, 2011.

[22] B. Ramakrishnan, R. B. Nishanth, M. M. Joe, and R. S. Shaji, "Comprehensive analysis of Highway, Manhattan and Freeway mobility models for vehicular ad hoc network", International Journal of Wireless and Mobile Computing, Vol. 9, No. 1, pp. 78-89, 2015.

[23] J. H. Kwon, H. S. Chang, T. Shon, J. J. Jung, and E. J. Kim, "Neighbor stability-based VANET clustering for urban vehicular environments", The Journal of Supercomputing, Vol. 72, No. 1, pp. 161-176, 2016.
[24] E. Gurumoorthi, and A. Ayyasamy, "Cache agent based location aided routing using distance and direction for performance enhancement in VANET", Telecommunication Systems, Vol. 73, No. 3, pp. 419-432, 2020.

[25] T. Lu, S. Chang, and W. Li, "Fog computing enabling geographic routing for urban area vehicular network", Peer-to-Peer Networking and Applications, Vol. 11, No. 4, pp. 749-755, 2018.

[26] K. K. Rana, S. Tripathi, and R. S. Raw, "Analytical Analysis of Improved Directional Location Added Routing Protocol for VANETS", Wireless Personal Communications, Vol. 98, No. 2, pp. 2403-2426, 2018.

[27] I. A. Abbasi, A. S. Khan, and S. Ali, "A Reliable Path Selection and Packet Forwarding Routing Protocol for Vehicular Ad hoc Networks", EURASIP Journal on Wireless Communications and Networking, Vol. 2018, No. 1, p. 236, 2018.

[28] S. Rahimi and M. A. J. Jamali, "A hybrid geographic-DTN routing protocol based on fuzzy logic in vehicular ad hoc networks", Peerto-Peer Networking and Applications, Vol. 12, No. 1, pp. 88-101, 2019.

[29] A. N. Hassan, A. H. Abdullah, O. Kaiwartya, Y. Cao, and D. K. Sheet, "Multi-metric geographic routing for vehicular ad hoc networks", Wireless Networks, Vol. 24, No. 7, pp. 2763-2779, 2018.

[30] O. Alzamzami and I. Mahgoub, "Fuzzy LogicBased Geographic Routing for Urban Vehicular Networks Using Link Quality and Achievable Throughput Estimations", IEEE Transactions on Intelligent Transportation Systems, Vol. 20, No. 6, pp. 2289-2300, 2019.

[31] L. Liu, C. Chen, Z. Ren, and C. Shi, "A link transmission-quality based geographic routing in Urban VANETs", In: Proc. of IEEE 28th Annual International Symposium on Personal, Indoor, and Mobile Radio Communications (PIMRC), Montreal, QC, pp. 1-6, 2017.

[32] N. Mantas, M. Louta, K. Katsaros, and S. Kraounakis, "Social CLWPR: A socially enhanced position-based routing protocol for handling misbehaviour in VANETs", In: Proc. of 8th International Conf. on Information, Intelligence, Systems \& Applications (IISA), Larnaca, pp. 1-6, 2017.

[33] R. K. Jaiswal and C. D. Jaidhar, "PPRP: Predicted Position Based Routing Protocol Using Kalman Filter for Vehicular Ad-hoc Network", In: Proc. of the 18th International Conf. on Distributed Computing and Networking - ICDCN '17, Hyderabad, India, No. 23, pp. 1-8, 2017. 
[34] K. Pandey, S. K. Raina, and R. S. Raw, "Distance and direction-based location aided multi-hop routing protocol for vehicular ad-hoc networks", International Journal of Communication Networks and Distributed Systems, Vol. 16, No. 1, pp. 71, 2016.

[35] O. Kaiwartya, and S. Kumar, "Cache agentbased geocasting in VANETs", International Journal of Information and Communication Technology, Vol. 7, No. 6, p. 562, 2015.

[36] Z. Qin, M. Bai, and D. Ralescu, "A fuzzy control system with application to production planning problems", Information Sciences, Vol. 181, No. 5, pp. 1018-1027, 2011.

[37] W. Su, S. J. Lee, and M. Gerla, "Mobility prediction and routing in ad hoc wireless networks", International Journal of Network Management, Vol. 11, No. 1, pp. 3-30, 2001. 